\title{
Social Media and Healthcare Technology Minitrack
}

\author{
Beth C Bock \\ Brown University Medical School \\ Beth_Bock@Brown.edu
}

\author{
Rochelle Rosen \\ Brown University Medical School \\ Rochelle_Rosen@Brown.edu
}

\begin{abstract}
The Social Media and Healthcare Technology mini-track addresses the emerging and increasingly broad range of social media use within healthcare; papers in the track provide insight into methodological, conceptual, and research design issues.
\end{abstract}

\section{Introduction}

Social media is changing the way healthcare organizations, consumers, and practitioners interact. Initially, many healthcare organizations avoided the use of social media, and even restricted their employees from using it. However, increasingly healthcare providers and organizations are realizing that social media provides specific opportunities to serve the public, patients and physicians, while also building awareness and enhancing their brand. Consumers are increasingly using social media to understand medical conditions and make health decisions, including selecting a doctor, researching courses of treatment, and finding communities composed of those with similar diagnoses. Physicians have also begun using social media to network professionally with colleagues and to share medical knowledge, both within the medical community and with patients. Clinicians and researchers have begun using social media to design and implement behavioral interventions for a variety of health conditions.

The objective of this new mini-track is to present current research examining the uses of social media in the delivery, receipt of, and communication about healthcare information and services. The six papers in the track include studies that: (1) reflect on patient and medical community use of social media, (2) evaluate the design, implementation and analysis of research conducted on or through social media applications; and (3) assess the impact of these platforms on patients, healthcare providers, organizations, and society in general.

The paper by Pagoto et al addresses the issue of incentivizing social media participation in a research context as a means of increasing engagement in an online social network weight loss program. This study examines user engagement within two Facebookdelivered weight loss interventions. Facebook is also explored in Farzan and Jonassaint's inquiry into peer leadership strategies among two groups of patients with Sickle Cell Disease. Understanding how organic and engineered leadership in social networks affects other participants is important for researchers and clinicians hoping to use social media to engage research participants and patients.

Two of the papers in the track explore the use of Twitter as a source of data and research content. Hemlsey et al outline technical methods used in an analysis of tweets tagged with Motor Neuron Disease and Amyotrophic Lateral Sclerosis hashtags. Chai et at conducted a tweet chat focus group in which participants directly address their willingness to provide research data through social media. Together these two papers inform the design and analysis of social media research through the twitter platform.

As social medial platforms and their use evolve, understanding whether and how information shared through them affects social norms becomes important for understanding both behavior and behavior change campaigns. Jian Rui's paper compares how exposure to drinking information on social network sites is related to perceived drinking norms among college students.

Finally, Gamache-OLeary and Grant provide an elegant review of social media literature which incorporates information seeking theory, virtual communities and social theory. They use feedback and advice from cancer patients to develop four key points for assessing how social media platform use in the medical setting influences patient and provider satisfaction.

The papers in this new track are evidence that, in its $50^{\text {th }}$ anniversary year, HICSS continues to address emerging and important topics in information technology and health care. 